\section{Perfil socioeconômico das populações expostas a resíduos da exploração de petróleo}

\section{A socio-economic profile of exposed populations to petroleum exploration residues}

Gyselle Guimarães Corrêa'

Thainá Alves Malhão'

\section{Carmen Ildes Rodrigues Fróes Asmus'}

Cláudia Medina Coeli'

'Instituto de Estudos em Saúde Coletiva da Universidade Federal do Rio de Janeiro - Rio de Janeiro (RJ), Brasil

Trabalho realizado no Instituto de Estudos em Saúde Coletiva da Universidade Federal do Rio de Janeiro - Rio de Janeiro (RJ), Brasil.

Fonte de financiamento: Conselho Nacional de Desenvolvimento Científico e Tecnológico (CNPq). Processo: 08/2007.

Correspondência: Gyselle Guimarães Corrêa Nogueira - Rua Benjamin Constant, 71, apto 207 Largo do Barradas - CEP: 24110-002 - Niterói (RJ), Brasil - E-mail: gy.correa@gmail.com

Conflito de interesse: nada a declarar.

\section{Resumo}

Introdução: A Macrorregião Ambiental Cinco (MRA-5) está situada no Rio de Janeiro. Seus municípios vêm sofrendo as consequências da acelerada urbanização, especialmente após a descoberta de grandes reservas de petróleo e gás natural (PGN) na Bacia de Campos. Objetivo: Descrever o perfil socioeconômico dessa região e comparar os seus indicadores no espaço e no tempo. Métodos: Trata-se de um estudo ecológico, onde se realizaram comparações entre os 11 municípios da MRA-5, baseadas no comportamento dos indicadores antes e após o incremento dos royalties e participações especiais (RPE); e na divisão das zonas de produção de PGN (zona de produção principal - ZPP - e zona limítrofe à ZPP - ZLZPP). O período de análise dos indicadores variou de 1991 a 2005. Resultados: Valores mais elevados do Produto Interno Bruto per capita foram encontrados nos municípios da ZPP, especialmente Macaé. O mesmo ocorreu nos RPE per capita, destacando-se Rio das Ostras (maior valor) e Nova Friburgo (menor valor). Em 2004, mais de $50 \%$ das receitas totais dos municípios eram dependentes dos recursos provenientes dos royalties. Em 2000, Nova Friburgo apresentou o melhor Índice de Desenvolvimento Humano. Quanto ao Índice de Qualidade dos Municípios Potencial para o Desenvolvimento, o maior valor foi encontrado em Macaé. Os maiores Índices de Exclusão Social foram encontrados em Macaé, Nova Friburgo e Rio das Ostras. Conclusão: Verificou-se, nos municípios da ZPP, um crescimento econômico excludente com grande dependência dos royalties. Deve-se investir em atividades econômicas alternativas para que não haja prejuízos à população com o término desses recursos.

Palavras-chave: petróleo; indicadores econômicos; indicadores de desenvolvimento; indicadores sociais; bacias hidrográficas; exploração de recursos naturais. 


\section{Abstract}

Introduction: Environmental Macro-Area Five (EMA-5) is located in Rio de Janeiro. Their municipalities are suffering the consequences of rapid urbanization, especially after the discovery of large reserves of petroleum and natural gas (PNG) in the Campos Basin. Objective: To describe the socioeconomic profile of the region and compare their indicators in space and time. Methods: This is an ecological study, which made comparisons between the 11 municipalities of EMA-5. It is based on the behavior of the indicators before and after the increase in royalties and special participatioms (RPE) and the division of the production areas of PNG (main production area - MPA and the border zone of MPA, BZMPA). The period of analysis of the indicators ranged from 1991 to 2005. Results: Higher values of gross domestic product per capita were found in the municipalities of MPA, especially Macaé. The same occurred in the RPE per capita, especially Rio das Ostras (higher value) and Nova Friburgo (lower value). In 2004 , more than $50 \%$ of total revenues of municipalities were dependent on the funds derived from royalties. In 2000, Nova Friburgo had the best Human Development Index. The highest value of the Quality Index of Municipalities Potential for Development was found in Macaé. The major indexes of Social Exclusion was found in Macaé, Nova Friburgo and Rio das Ostras. Conclusion: It was found, in the municipalities of MPA, economic growth with great exclusive reliance on royalties. It should invest in alternative economic activities for which there is no damage to the population with the completion of these resources.

Keywords: petroleum; economic indexes, development indicators; social indicators; hydrographic basins; natural resources exploitation.

\section{Introdução}

O processo de industrialização mundial, ocorrido no século XX, consolidou o petróleo como principal fonte de energia. A partir da Segunda Guerra Mundial, o petróleo se estabeleceu como o paradigma energético mundial ${ }^{1}$. Atualmente, o petróleo é de fundamental importância para a sociedade pois, além do fornecimento de energia e de seus derivados para a manufatura de bens de consumo ${ }^{2}$, tem um efeito significativo à produtividade econômica e no bem-estar da população ${ }^{3}$.

O mundo tem se tornado cada vez mais dependente do petróleo para o seu progresso econômico. Na condição de recurso natural não renovável, o petróleo é finito, e torna-se cada vez mais escasso em função da demanda crescente de energia no mundo ${ }^{1}$. O setor petrolífero apresenta forte influência na economia, tanto pelo impacto multiplicador sobre as demais cadeias produtivas, quanto por seus efeitos sobre o balanço de pagamentos e contas públicas ${ }^{4}$. Traz como resultados, o progresso tecnológico, e o desenvolvimento social. Entretanto, devem ser considerados os reflexos na população e no meio ambiente, decorrentes dessa atividade.

A atividade de exploração e produção (E\&P) de petróleo e gás no ambiente marítimo cresce a cada ano, e a evolução no desenvolvimento tecnológico permite a exploração de cada vez mais reservas, em águas profundas e ultraprofundas ${ }^{5}$.

A distribuição das participações governamentais derivadas da exploração, desenvolvimento e produção do petróleo e do gás natural é regida pela Lei 9.478/97. O assunto é coordenado pelo Conselho Nacional de Política Energética e pela Agência Nacional do Petróleo (ANP). Envolve o pagamento das seguintes participações governamentais: bônus de assinatura, royalties, participação especial e pagamento pela ocupação ou retenção de área.

A partir da data de início da produção de cada campo, o volume e a qualidade do petróleo e gás natural produzidos 
são determinados periódica e regularmente nos pontos de medição da produção, por conta e risco do concessionário, com a utilização dos métodos, equipamentos e instrumentos de medição previstos no respectivo plano de desenvolvimento e observadas as regras específicas emanadas da ANP ${ }^{6}$.

Por esta exploração e produção dentro dos limites do seu mar territorial, os municípios fluminenses que integram essa região recebem uma compensação financeira denominada royalties. Sua distribuição é feita em consonância com a lei ${ }^{\circ}$ 9478/977 . Além desse benefício, essas localidades ainda contam com as participações especiais (PE) devidas pelos concessionários nos casos de grande volume de produção ou grande rentabilidade ${ }^{8}$.

A distribuição dos recursos para os municípios toma por base a divisão das zonas de produção de petróleo e gás realizada pela $\mathrm{ANP}^{7}$. A Zona de produção principal (ZPP) é formada pelo conjunto dos municípios onde se localizam três ou mais instalações dos seguintes tipos: instalações industriais para processamento, tratamento, armazenamento e escoamento, excluindo-se os dutos e àquelas relacionadas às atividades de apoio à exploração, produção e escoamento (portos, aeroportos, oficinas de manutenção e fabricação, almoxarifados, armazéns e escritórios). A zona de produção secundária (ZPS) contempla os municípios atravessados por oleodutos ou gasodutos, incluindo as respectivas estações de compressão e de bombeio, destinadas exclusivamente ao escoamento da produção petrolífera marítima. Por fim, a zona limítrofe à zona de produção principal (ZLZPP) compreende os municípios contíguos àqueles que integram a $\mathrm{ZPP}$, bem como os que sofrem consequências sociais ou econômicas da produção ou exploração do petróleo e gás?

De acordo com o Artigo $9^{\circ}$ da Lei 7990/89, os Estados devem transferir aos municípios $25 \%$ dos royalties repassados à Unidade Federada. Aos municípios que integram a ZPP, são destinados $60 \%$ desse montante; aos municípios da ZPS, $10 \%$ e aos municípios da ZLZPP, 30\%. Além desses recursos, os municípios recebem $80 \%$ do Fundo Especial ( $10 \%$ dos royalties).

Por ser de grande valor energético, as atividades de exploração e produção de petróleo são intensas ${ }^{9}$. O consumo ilimitado desse recurso acelera a degradação do meio ambiente, levando ao esgotamento de recursos naturais, gerando impactos importantes para a sobrevivência do planeta, dentre eles encontra-se a liberação desenfreada de carbono fóssil para a atmosfera e o aquecimento global. Vale lembrar que a palavra poluição está quase sempre relacionada às atividades de exploração e produção de petróleo, principalmente quando ocorrem vazamentos de óleos e alterações em ecossistemas marinhos, costeiros e terrestres ${ }^{10}$. No entanto, tais atividades alteram a dinâmica socioambiental dos locais onde se instalam; portanto, é possível encontrar impactos oriundos de processos de adensamento urbano, como alterações na qualidade do ar e lançamento de esgotos.

Cabe ainda ressaltar que o impacto da produção e exploração de petróleo não afeta somente ao meio ambiente, já que estudos comprovam os constituintes do petróleo, dentre eles os hidrocarbonetos policíclicos aromáticos, em seu conjunto, como extremamente tóxicos aos seres humanos, sendo muitos classificados como cancerígenos, mutagênicos e teratogênicos ${ }^{11}$. As atividades desenvolvidas pela indústria petrolífera estão além do desempenho de suas unidades de operação, aparentemente isoladas em alto mar, ou da importância de seus derivados para o consumo. Essa atividade pode interferir nas dinâmicas socioespaciais do lugar em que está estabelecida ou em qualquer parte do globo. Partindo desse pressuposto, a Macrorregião Ambiental Cinco (MRA-5) é uma área situada no Estado do Rio de Janeiro (RJ), compreendendo os municípios de Carapebus, Campos dos Goytacazes, Casimiro de Abreu, Conceição de Macabu, Macaé, Nova Friburgo, Quissamã, Rio das Ostras, 
Santa Maria Madalena, São João da Barra e Trajano de Moraes. Esses vêm sofrendo as consequências de um acelerado processo de urbanização, especialmente a partir da década de 1970, com a descoberta de grandes reservas de petróleo e gás natural na Bacia de Campos ${ }^{12}$.

Dessa forma, o presente artigo tem como objetivos descrever o perfil socioeconômico da população residente nos municípios da MRA-5 e comparar os seus indicadores no espaço e no tempo, buscando, dessa forma, avaliar os impactos potenciais da exploração de petróleo nessa região.

\section{Materiais e métodos}

Foi realizado um estudo ecológico, em que foram analisados indicadores socioeconômicos, empregando-se três eixos de comparação: (1) entre os municípios da MRA-5 e desses com a mediana do Estado do RJ; (2) baseada no comportamento dos indicadores num período anterior e posterior ao incremento das receitas, oriundas de participações governamentais; (3) e de acordo com a divisão das zonas de produção de petróleo e gás, apresentado anteriormente.

Com o objetivo de verificar a influência das participações governamentais nas receitas totais municipais (soma das receitas correntes e receitas de capital), foi calculado o indicador royalties versus receitas totais. Para perceber as disparidades existentes entre as unidades de análise, também foram construídos os índices royalties e participações especiais (RPE) per capita e produto interno bruto (PIB) per capita. Esses três indicadores foram empregados para a avaliação da dimensão econômica. Além disso, para avaliar o comportamento desses indicadores, no período de referência, foram calculadas as taxas de expansão $\{[$ (valor no período inicial*100)/valor no período final] -100$\}^{8}$. Com o objetivo de obter um maior conhecimento sobre a região estudada, os índices compostos selecionados para o estudo foram: índice de desenvolvimento humano municipal (IDH-M), índice de exclusão social (IES) e os índices de qualidade dos municípios: potencial para o desenvolvimento (IQM-PD); sustentabilidade fiscal (IQM-SF); carências (IQM-C) ${ }^{13-16}$. Esses índices permitem capturar uma dada distância entre a realidade existente e o desenho de uma sociedade ideal, em que se presencie alto grau de equidade e cidadania. A matriz teórica e fonte de dados desses indicadores podem ser observadas no Quadro 1. Os anos estudados nos dois períodos de referência (anterior e posterior ao incremento das receitas) variaram conforme o tipo de indicador avaliado e da sua disponibilidade de dados. Na análise dos indicadores econômicos, foram selecionados os anos de 1997 a 2004. No entanto, nos índices compostos, não houve um período pré-estabelecido.

De acordo com Pacheco (2003) ${ }^{4}$, apesar das modificações inseridas na Lei do Petróleo datarem de 1997, o impacto no recolhimento dos royalties só foi expressivo a partir de 1998. De maneira semelhante, as participações especiais só se tornaram relevantes a partir do ano de 2000 , ou seja, no início da exploração e produção dos campos gigantes, na Bacia de Campos. Portanto, o corte temporal escolhido para análise permitiu que houvesse um tempo mínimo de indução dos efeitos da exploração de petróleo, bem como das receitas advindas deste processo sobre as condições socioeconômicas da população estudada. Já para os indicadores compostos, o período foi determinado pela disponibilidade de dados no momento em que a análise foi concluída. No que se refere às populações utilizadas para o cálculo dos indicadores econômicos, foi resgatada a base de dados fornecida pelo Instituto Brasileiro de Geografia e Estatísticas (IBGE), com base nos Censos Demográficos de 1991 e 2000 e utilizando-se o método de Lagrange nos cálculos das populações em $1^{\circ}$ de julho para os anos intercensitários ${ }^{17}$. 
Quadro 1. Quadro resumo da matriz teórica dos índices compostos

Chart 1. Summary table of the theoretical framework of the composite indices

\begin{tabular}{|c|c|c|}
\hline Indicadores & Conceituação & Fonte \\
\hline $\begin{array}{l}\text { Índice de desenvol- } \\
\text { vimento humano } \\
\text { municipal }\end{array}$ & $\begin{array}{l}\text { É obtido pela média aritmética simples de três sub-índices, referentes às dimensões } \\
\text { Longevidade, Educação e Renda. Quanto mais próximo de } 1,000 \text { o valor deste } \\
\text { indicador, maior o nível de desenvolvimento humano da região. Baixo desenvolvi- } \\
\text { mento humano: até } 0,499 ; \text { Médio: entre } 0,500 \text { e } 0,799 \text {; Alto: }>0,800 \text {. }\end{array}$ & 1 \\
\hline $\begin{array}{l}\text { Índice de qualidade dos } \\
\text { municípios - potencial } \\
\text { para o desenvolvimento }\end{array}$ & $\begin{array}{l}\text { Procura sintetizar os vários aspectos das condições básicas necessárias ao investi- } \\
\text { mento produtivo. Varia de } 0,000 \text { a 1,000, ou seja, do pior para o melhor potencial } \\
\text { para o desenvolvimento. }\end{array}$ & 2 \\
\hline $\begin{array}{l}\text { Índice de qualidade dos } \\
\text { municípios - sustentabi- } \\
\text { lidade fiscal }\end{array}$ & $\begin{array}{l}\text { Pretende avaliar a situação fiscal dos municípios, com base em indicadores finan- } \\
\text { ceiros e fiscais. Alta sustentabilidade fiscal: acima de 0,782; média superior: de 0,491 } \\
\text { a 0,782; média: de 0,333 a 0,490; média inferior: de 0,226 a 0,332; baixa: de 0,185 a } \\
0,225 \text { e muito baixa: abaixo de 0,185. }\end{array}$ & 3 \\
\hline $\begin{array}{l}\text { Índice de qualidade dos } \\
\text { municípios - carências }\end{array}$ & $\begin{array}{l}\text { Busca medir as carências representadas não pela pobreza, mas pela graduação da } \\
\text { qualidade de vida em educação; saúde; habitação e saneamento; mercado de trabalho; } \\
\text { comércio; segurança; esporte, cultura e lazer; descentralização administrativa; comuni- } \\
\text { cações; participação comunitária e transportes. Município carente: >44\% }\end{array}$ & 4 \\
\hline Índice de exclusão social & $\begin{array}{l}\text { É construído com base no índice de pobreza, índice de emprego, índice de } \\
\text { desigualdade, índice de anos de estudo, índice de alfabetização, índice de concen- } \\
\text { tração de jovens e índice de violência. As piores condições de vida equivalem a } \\
\text { valores próximos a zero, enquanto as melhores situações sociais estão próximas a um. }\end{array}$ & 5 \\
\hline
\end{tabular}

Fontes:

1. PNUD - Atlas do Desenvolvimento Humano no Brasil, 2000.

2. CIDE-RJ - Índice de Qualidade dos Municípios Potencial para o Desenvolvimento II (CD).

3. CIDE-RJ - Índice de Qualidade dos Municípios Sustentabilidade Fiscal (CD).

4. CIDE-RJ - Índice de Qualidade dos Municípios Carências.

5. Pochmann - Atlas da Exclusão Social no Brasil.

Sources:

1. UNDP - Atlas of Human Development in Brazil, 2000.

2. DICE-RJ - Quality Index Municipal Development Potential II (CD).

3. DICE - RJ - Quality Index Municipal Fiscal Sustainability (CD).

4. DICE - RJ - Quality Index Municipal Needs.

5. Pochmann - Atlas of Social Exclusion in Brazil.

\section{Resultados}

\section{Indicadores econômicos}

Por meio da análise da Tabela 1, foi possível perceber as disparidades econômicas existentes na MRA-5. De forma geral, na Figura 1, verificou-se que as medianas dos municípios da ZPP foram superiores às da ZLZPP e às estaduais, tanto no que se refere aos valores dos três indicadores econômicos selecionados, como nas suas taxas de expansão. Macaé, conhecida como a cidade do petróleo, além de apresentar os maiores valores de PIB per capita, em todo período avaliado, também teve a maior taxa de variação de 1997 a 2004 (305,4\%).

Rio das Ostras e Quissamã, ao contrário de Nova Friburgo, apresentaram as maiores arrecadações de RPE per capita, sendo que o maior e menor crescimento médio, entre 1997 e 2004, foi encontrado em Campos dos Goytacazes (930,8\%) e Carapebus $(263,4 \%)$, respectivamente. Outro fato que mereceu atenção, foi o importante decréscimo nessas participações governamentais no município de São João da Barra, entre 2001 e 2002.

Todos os municípios da ZPP apresentaram um forte grau de dependência em relação à Bacia de Campos. As compensações financeiras provenientes da atividade petrolífera representaram, na maioria dessas localidades, mais da metade das receitas totais. Esse comportamento é contrário ao notado na ZLZPP, que apresentou maior autonomia financeira. De todos os municípios da MRA-5, Nova Friburgo foi o 
Tabela 1. Indicadores econômicos dos municípios da Macrorregião Ambiental Cinco e do Estado do Rio de Janeiro, nos anos de 1997 a 2004

Table 1. Economic indicators of the municipalities of Environmental Macro-Area Five (EMA-5) and the state of Rio de Janeiro, in 1997 to 2004

\begin{tabular}{|c|c|c|c|c|c|c|c|c|c|c|}
\hline Indicador & Municípios & 1997 & 1998 & 1999 & 2000 & 2001 & 2002 & 2003 & 2004 & $\Delta$ 1997-2004 (\%) \\
\hline \multirow{11}{*}{ PIB per capita (1) } & Quissamã & 4165,9 & 3140,8 & 3975,3 & 4978,2 & 5476,1 & 6844,6 & 8011,8 & 8829,1 & 111,9 \\
\hline & Carapebus & 3600,3 & 3071,0 & 3116,5 & 3421,0 & 3736,9 & 4578,6 & 4352,5 & 4750,1 & 31,9 \\
\hline & Macaé & 6535,7 & 7237,7 & 7683,4 & 8545,6 & 13464,6 & 20388,1 & 23082,4 & 26494,3 & 305,4 \\
\hline & Rio das Ostras & 4997,0 & 3523,9 & 3922,5 & 4189,1 & 4450,0 & 5707,9 & 9217,7 & 9752,9 & 95,2 \\
\hline & Casimiro de Abreu & 3334,1 & 4761,6 & 4554,5 & 5530,5 & 5744,9 & 7793,1 & 8708,3 & 10114,5 & 203,4 \\
\hline & São|João da Barra & 4552,5 & 2839,5 & 3201,1 & 3427,8 & 3428,6 & 4470,4 & 5235,2 & 5630,5 & 23,7 \\
\hline & Compos dos Goytacazes & 3703,1 & 4274,7 & 4263,1 & 4277,1 & 4630,5 & 5153,5 & 6816,6 & 7564,0 & 104,3 \\
\hline & Cardoso Moreira & 3032,5 & 2546,6 & 2546,7 & 2925,5 & 3521,8 & 3747,8 & 4784,5 & 5049,7 & 66,5 \\
\hline & Santa Maria Madalena & 3019,0 & 3239,7 & 2857,6 & 3195,5 & 3727,3 & 4876,5 & 5798,1 & 5804,8 & 92,3 \\
\hline & Trajano de Moraes & 2499,3 & 2722,7 & 2897,0 & 3636,5 & 3480,0 & 4814,5 & 6823,9 & 6602,0 & 164,1 \\
\hline & Nova Friburgo & 4763,5 & 5563,9 & 5441,8 & 5849,3 & 5591,0 & 6310,8 & 8104,6 & 8443,2 & 77,2 \\
\hline Indicador & Municípios & 1997 & 1998 & 1999 & 2000 & 2001 & 2002 & 2003 & 2004 & $\Delta$ 1997-2004 (\%) \\
\hline \multirow{11}{*}{$\begin{array}{l}\text { Royalties e partici- } \\
\text { pações especiais } \\
\text { per capita ( } 2 \text { ) }\end{array}$} & Quissamã & - & - & 1072,2 & 2300,7 & 2474,7 & 3322,6 & 4402,9 & 4447,8 & 314,9 \\
\hline & Carapebus & - & - & 687,2 & 1340,8 & 1520,0 & 2269,7 & 2594,3 & 2497,4 & 263,4 \\
\hline & Macaé & - & - & 278,3 & 647,0 & 844,2 & 1311,0 & 1831,7 & 1980,7 & 611,6 \\
\hline & Rio das Ostras & - & - & 552,9 & 1701,6 & 2364,2 & 3439,8 & 4976,9 & 4881,7 & 782,9 \\
\hline & Casimiro de Abreu & - & - & 272,7 & 533,5 & 634,6 & 1108,7 & 1391,1 & 1295,1 & 375,0 \\
\hline & São|João da Barra & - & - & 183,8 & 450,3 & 561,9 & 202,4 & 1076,7 & 1341,4 & 629,7 \\
\hline & Compos dos Goytacazes & - & - & 122,6 & 369,5 & 504,1 & 741,0 & 1175,7 & 1263,9 & 930,8 \\
\hline & Cardoso Moreira & - & - & 46,5 & 95,1 & 109,0 & 162,9 & 213,5 & 184,2 & 296,4 \\
\hline & Santa Maria Madalena & - & - & 71,5 & 149,8 & 168,5 & 264,0 & 346,4 & 307,9 & 330,8 \\
\hline & Trajano de Moraes & - & - & 72,6 & 154,6 & 173,9 & 273,3 & 359,2 & 317,8 & 337,8 \\
\hline & Nova Friburgo & - & - & 8,9 & 18,7 & 19,0 & 31,7 & 41,3 & 36,7 & 311,8 \\
\hline Indicador & Municípios & 1997 & 1998 & 1999 & 2000 & 2001 & 2002 & 2003 & 2004 & $\Delta$ 1997-2004 (\%) \\
\hline \multirow{11}{*}{$\begin{array}{l}\text { Royalties vs } \\
\text { Receitas totais (3) }\end{array}$} & Quissamã & - & - & 47,1 & 58,8 & 49,9 & 51,1 & 53,4 & 55,5 & - \\
\hline & Carapebus & - & - & 44,4 & 54,1 & 51,4 & 53,7 & 58,4 & 56,1 & - \\
\hline & Macaé & - & - & 37,7 & 52,6 & 50,0 & 53,7 & 51,5 & 54,7 & - \\
\hline & Rio das Ostras & - & - & 45,6 & 71,5 & 67,9 & 64,9 & 63,6 & 66,7 & - \\
\hline & Casimiro de Abreu & - & - & 24,9 & 37,1 & 35,0 & 44,1 & 48,2 & 45,7 & - \\
\hline & São|João da Barra & - & - & 31,1 & 44,0 & 42,3 & 21,0 & 51,5 & 59,2 & - \\
\hline & Compos dos Goytacazes & - & - & 31,4 & 55,4 & 54,3 & 58,5 & 67,3 & 66,7 & - \\
\hline & Cardoso Moreira & - & - & 11,8 & 16,0 & 13,6 & 15,4 & 22,0 & 19,2 & - \\
\hline & Santa Maria Madalena & - & - & 8,0 & 13,2 & 13,3 & 20,1 & 23,0 & 18,7 & - \\
\hline & Trajano de Moraes & - & - & 9,0 & 14,6 & 14,8 & 19,5 & 26,2 & 21,6 & - \\
\hline & Nova Friburgo & - & - & 2,2 & 4,3 & 4,3 & 6,4 & 8,2 & 6,1 & - \\
\hline
\end{tabular}

Nota: (1) Produto interno bruto per capita (em milhões de reais); (2) Royalties e participações especiais per capita (em mil reais); (3) Royalties $x$ Receitas totais (em \%); $\Delta$ variação. Fonte: CIDE/ RJ, 1997 - 2004

Note: (1) Gross domestic product per capita (million dollars); (2) Royalties and special participation per capita (in thousand reais); (3) Royalties x Total Revenues (in\%); $\Delta$ variation Source: CIDE/RJ, 1997-2004.

único que apresentou percentuais de royalties $v$ s. receitas totais inferiores a mediana estadual.

\section{Índices Compostos}

De acordo com a Tabela 2, no que tange ao IDH-M, constatou-se que todos os municípios melhoraram seus índices. Além disso, dentro da ZPP, Macaé apresentou o melhor desempenho, seguido por
Casimiro de Abreu. Na análise da ZLZPP, Nova Friburgo e Conceição de Macabu obtiveram os maiores valores.

Quanto ao IQM-PD, observaram-se algumas discrepâncias entre as zonas de produção de petróleo e gás. Por meio da Figura 2, notou-se que nos municípios da ZPP, a mediana variou de 0,333 (1998) a 0,459 (2005), enquanto que nos da ZLZPP, de 0,163 (1998) a 0,205 (2005). Isso pôde ser notado inclusive na análise intermunicipal, ao passo que Macaé 
PIB per capita

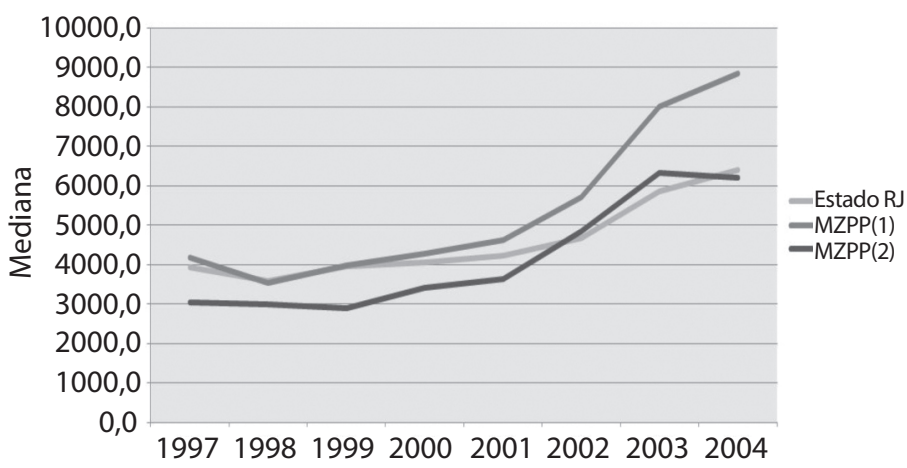

Royalties e participações especiais per capita

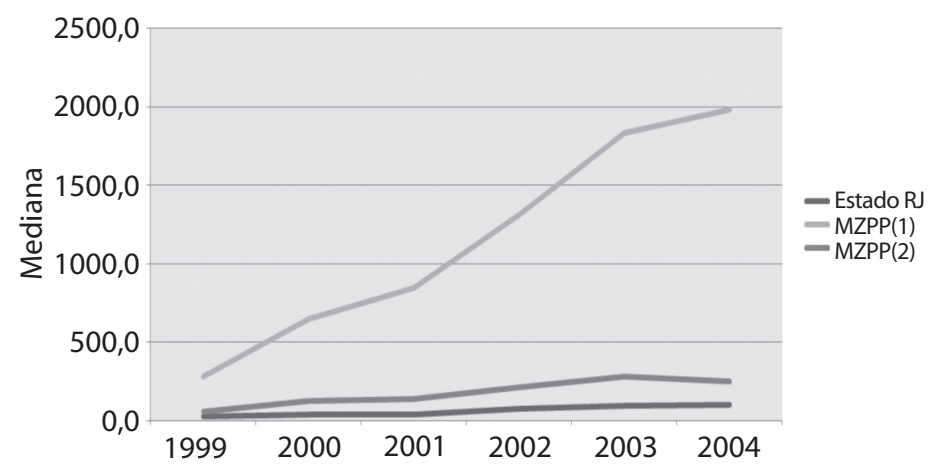

Royalties vs Receitas Totais

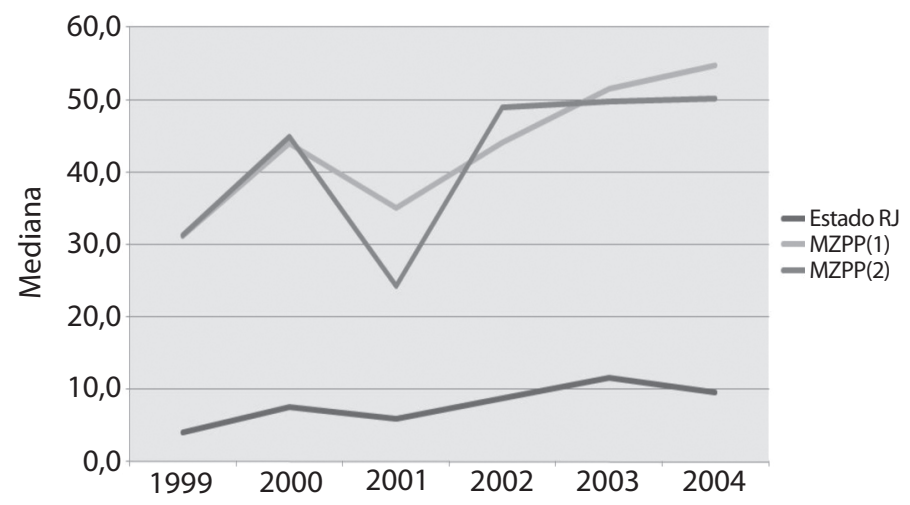

Nota: (1) Municípios da Zona de Produção Principal (MZPP); (2) Municípios da Zona limítrofe a Zona de Produção Principal (MLZPP).

Note: (1) Main production Zone Municipalities (MZPP); (2) Bordering the Main Production Zone Municipalities (MLZPP).

Figura 1. Indicadores econômicos dos municípios da Macrorregião Ambiental Cinco e do Estado do Rio de Janeiro, nos anos de 1997 a 2004 - Medianas do Estado do Rio de Janeiro, Municípios da Zona de Produção Principal e Municípios Limítrofes a Zona de Produção Principal Figure 1. Economic indicators of the municipalities of Environmental Macro-Area Five (EMA-5) and the state of Rio de Janeiro, in 1997 to 2004 - Medians of the State of Rio de Janeiro, Municipalities Main Production Zone and Municipalities Bordering the Main Production Zone apresentou o melhor potencial para o desenvolvimento e Trajano de Moraes, o pior.

No que tange ao IQM-SF, Quissamã $(1,000)$ e Carapebus $(0,819)$ tiveram uma alta sustentabilidade fiscal. No outro extremo, encontrou-se Conceição de Macabu $(0,194)$, que se caracterizou por um baixo desempenho. O único município da ZPP que apresentou sustentabilidade fiscal inferior à mediana dos da ZLZPP $(0,313)$ e do Estado $(0,333)$ foi São João da Barra $(0,253)$.

De acordo com o IQM-C, os únicos municípios que conseguiram ultrapassar o primeiro nível da pirâmide (o das necessidades básicas) foram Macaé $(41,9 \%)$ e Campos dos Goytacazes (42,3\%). Os demais se encontravam em estado de carência.

Ao considerar o fenômeno da exclusão social, foram observadas as melhores condições sociais em Macaé e em Nova Friburgo, e as piores, em Trajano de Moraes, Conceição de Macabu e Quissamã.

\section{Discussão}

Neste estudo constatou-se, entre 1999 e 2004, um incremento na arrecadação e elevada dependência das participações governamentais provenientes da exploração e produção de petróleo e gás na Bacia de Campos. A partir desse panorama, foi possível perceber que o aporte de recursos oriundos da indústria de exploração e produção do petróleo não se distribuiu uniformemente entre os municípios que compõem as Zonas de Produção, a forte concentração desses em alguns municípios, combinado com as condições geográficas e sociais historicamente construídas, se expressa em graus de dependência econômica diferenciados ${ }^{18}$.

Esse resultado aponta para a necessidade de se monitorar criticamente a forma como os recursos provenientes dessa fonte são utilizados e fiscalizados, e examinar como se produzem as relações entre a administração pública e seus atores locais, buscando garantir o seu uso sustentável, uma vez que esses recursos naturais não são renováveis ${ }^{6,19}$. 
Tabela 2. Índices compostos dos municípios da Macrorregião Ambiental Cinco e do Estado do Rio de Janeiro Table 2. Composite indices of municipalities of Environmental Macro-Area Five (EMA-5) and the state of Rio de Janeiro

\begin{tabular}{|c|c|c|c|c|c|c|c|c|c|c|c|c|}
\hline & $\begin{array}{l}\text { Ano de } \\
\text { análise }\end{array}$ & Quissamã & Carapebus & Macaé & $\begin{array}{l}\text { Rio das } \\
\text { Ostras }\end{array}$ & $\begin{array}{l}\text { Casimiro } \\
\text { de Abreu }\end{array}$ & $\begin{array}{l}\text { São João } \\
\text { da Barra }\end{array}$ & $\begin{array}{c}\text { Campos dos } \\
\text { Goytacazes }\end{array}$ & $\begin{array}{l}\text { Cardoso } \\
\text { Moreira }\end{array}$ & $\begin{array}{c}\text { Santa Maria } \\
\text { Madalena }\end{array}$ & $\begin{array}{c}\text { Trajano de } \\
\text { Moraes }\end{array}$ & $\begin{array}{c}\text { Nova } \\
\text { Friburgo }\end{array}$ \\
\hline $\mathrm{IDH}-\mathrm{M}(1)$ & 2000 & 0,732 & 0,740 & 0,790 & 0,775 & 0,781 & 0,723 & 0,752 & 0,738 & 0,734 & 0,723 & 0,810 \\
\hline \multirow{2}{*}{ IQM-PD (2) } & 1998 & 0,231 & 0,149 & 0,479 & 0,333 & 0,471 & 0,151 & 0,425 & 0,252 & 0,070 & 0,073 & 0,309 \\
\hline & 2005 & 0,353 & 0,160 & 0,639 & 0,519 & 0,462 & 0,174 & 0,459 & 0,222 & 0,188 & 0,086 & 0,364 \\
\hline IQM-SF (3) & 1999 & 1,000 & 0,819 & 0,577 & 0,778 & 0,567 & 0,253 & 0,566 & 0,194 & 0,380 & 0,333 & 0,292 \\
\hline IQM-C (4) & 1991-2001 & 57,40 & 62,50 & 41,90 & 55,40 & 50,00 & 52,40 & 42,30 & 57,30 & 62,70 & 63,30 & 44,60 \\
\hline IES (5) & 2000 & 0,477 & 0,515 & 0,604 & 0,523 & 0,519 & 0,483 & 0,511 & 0,473 & 0,484 & 0,468 & 0,591 \\
\hline
\end{tabular}

Nota: (1) Índice de Desenvolvimento Humano Municipal (IDH-M). Fonte: PNUD, 2003; (2) Índice de Qualidade dos Municípios - Potencial para o Desenvolvimento (IQM-PD). Fonte: CIDE-RJ, 1998-2005; (3) Índice de Qualidade dos Municípios - Sustentabilidade Fiscal (IQM-SF). Fonte: CIDE-RJ, 1999; (4) Índice de Qualidade dos Municípios - Carência (IQM-C) (em \%). Fonte: CIDE-RJ, 1991-2001; (5) Índice de Exclusão Social (IES). Fonte: Pochmann et al, 2003. Note: (1) Human Development Index Municipal. Source (IDH-M): UNDP, 2003; (2) Quality Index of Municipalities - Potential for Development (IQM-PD). Source: CIDE-RJ, 1998-2005, (3) Quality Index of Municipalities - Fiscal Sustainability (IQM-SF). Source: CIDE-RJ, 1999; (4) Quality Index of Municipalities - Grace (IQM-C) (in\%). Source: CIDE-RJ, 1991-2001, (5) Social Exclusion Index (IES). Source: Pochmann et al, 2003
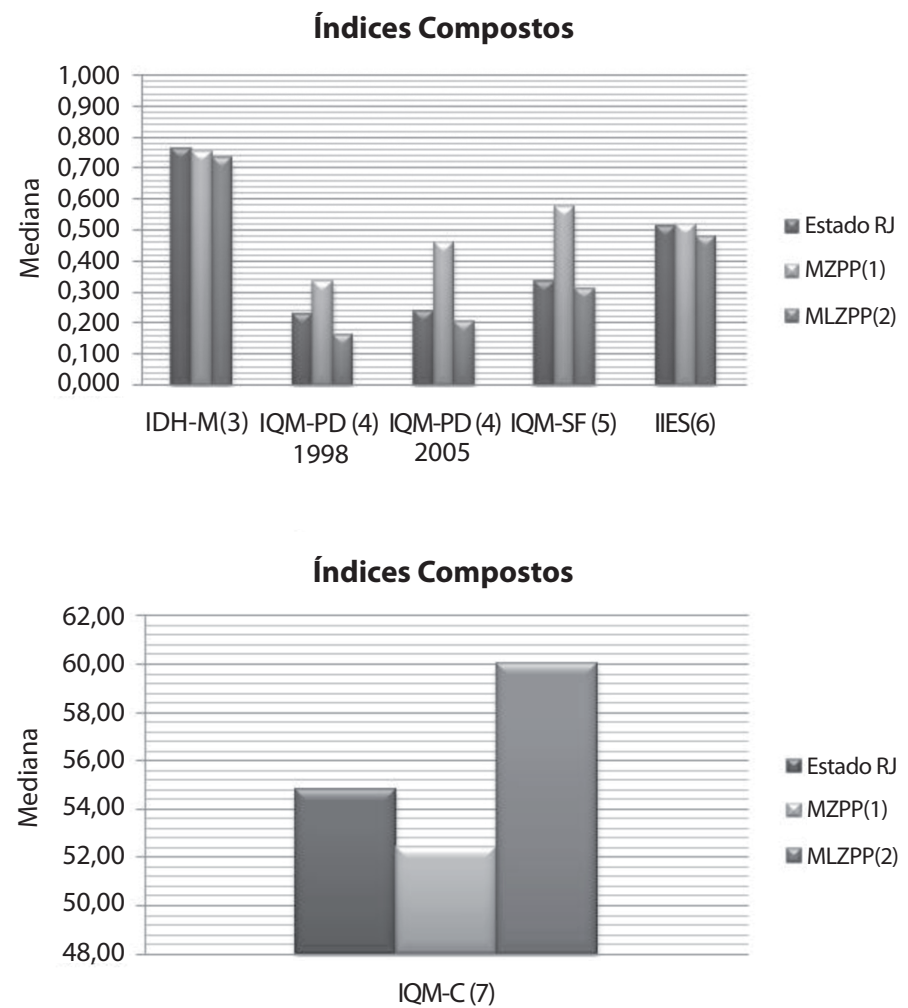

Nota: (1) Municípios da Zona de Produção Principal (MZPP); (2) Municípios da Zona limítrofe a Zona de Produção Princiapal (MLZPP); (3) Índice de Desenvolvimento Humano Municipal (IDH-M). Fonte: PNUD, 2003; (4) Índice de Qualidade dos Municípios - Potencial para o Desenvolvimento (IQM-PD). Fonte: CIDE-RJ, 1998-2005; (5) Índice de Qualidade dos Municípios - Sustentabilidade Fiscal (IQM-SF). Fonte: CIDE-RJ, 1999; (6) Índice de Exclusão Social (IES). Fonte: Pochmann et al, 2003. (7) Índice de Qualidade dos Municípios - Carência, em \% (IQM-C). Fonte: CIDE-RJ, 1991-2001.

Note: (1) Main production Zone (ZPP); (2) Bordering the Main Production Zone (LZPP); (3) Human Development Index Municipal (IDH-M). Source: UNDP, 2003; (4) Quality Index of Municipalities - Potential for Development (IQM-PD). Source: CIDE-RJ, 1998-2005, (5) Quality Index of Municipalities - Fiscal Sustainability (IQM-SF). Source: CIDE-RJ, 1999; (6) Social Exclusion Index (IES). Source: Pochmann et al, 2003; (7) Quality Index of Municipalities - Grace, in \% (IQM-C). Source: CIDE-RJ, 1991-2001.

Figura 2. Índices compostos dos municípios da Macrorregião Ambiental Cinco e do Estado do Rio de Janeiro Figure 2. Composite indices of municipalities of Environmental Macro-Area Five (EMA-5) and the state of Rio de Janeiro 
No entanto, no que rege a legislação referente aos recursos do petróleo, os administradores públicos possuem uma grande possibilidade de aplicação dos recursos, pois a lei não restringe os setores nos quais os royalties possam ser investidos. É imprescindível que as regras de rateio sejam revistas, já que o objetivo desse recurso é preparar os municípios impactados pelas atividades em questão para a fase pós-esgotamento das jazidas, pois o repasse acaba sendo feito de forma sobredimensionada $\mathrm{e}$ baseada em critérios de proximidade física que não consideram os princípios da justiça entre gerações ${ }^{18}$.

Através de um estudo realizado em três Estados brasileiros beneficiários dos royalties, no ano de 2007, focando nos respectivos municípios, o autor constatou que os municípios que receberam receitas de royalties a título de compensação por serem regiões produtoras de petróleo tiveram uma tendência a desempenhos inferiores em termos de crescimento do PIB. O mesmo resultado foi encontrado quando avaliada as receitas de royalties no interior dos três Estados. O autor destacou ainda que quanto maior o volume de royalties transferidos, menor tende a ser o crescimento econômico do município ${ }^{20}$.

A não diversificação produtiva, portanto, promove gargalos nos municípios, pois diminuem as suas possibilidades de alternativas econômicas ao fim da "indústria do petróleo". Ao contrário, começam a sofrer com a falta de investimentos em saneamento, habitação e urbanização, elementos hoje considerados internacionalmente como capazes de, em longo prazo, diminuir o fluxo turístico de maior renda inclusive internacional - pela perda de balneabilidade de suas praias e do aumento da degradação urbana ${ }^{21}$.

É importante que essas localidades promovam o desenvolvimento local, baseado nas potencialidades municipais. É necessário diversificar em vários setores da economia buscando, além da geração de empregos diretos, a atração de capital externo. A diversificação da economia permite que aumentem o número de empresas e de outros serviços, bem como a circulação de pessoas e de capital.

Usualmente o bem-estar de uma população e, consequentemente, a classificação de países ou regiões, é avaliado pelo tamanho do seu PIB per capita. Entretanto, o progresso humano e a evolução das condições de vida das pessoas não podem ser medidas apenas por aspectos econômi$\cos ^{22}$. Por isso, neste trabalho optou-se por empregar índices compostos, que representam medidas socioeconômicas mais abrangentes, incluindo outras dimensões fundamentais da vida e da condição humana.

Em relação ao IDH-M cabe salientar que, apesar de refletir as dimensões fundamentais da vida e da condição humana, o método utilizado para o cálculo desse indicador homogeneíza realidades distintas, colocando muitas vezes no mesmo patamar municípios abastados, que apresentam precárias condições sociais e municípios com melhores condições sociais apesar de serem relativamente pobres de acordo com o nível de renda per capita. $\mathrm{O}$ IDH-M impede, portanto, a identificação de municípios segundo suas carências de políticas sociais específicas. Essa limitação faz que o IDH-M não seja robusto para a seleção de municípios que serão alvo de políticas públicas, uma vez que municípios com mesmo IDH-M podem apresentar requerimentos de políticas sociais completamente distintas ${ }^{23}$. Esse indicador apenas sinaliza como os recursos são alocados nas áreas de saúde e educação, por exemplo, não incorporando a desigualdade na distribuição. Sob o aspecto da obtenção de dados, nem todos os países e regiões os têm disponíveis e quando os têm, são de qualidade duvidosa ${ }^{24}$.

Não se pode afirmar que as melhoras nesse índice foram resultados diretos dos royalties. Primeiro, porque esse não foi construído no sentido de avaliar as contribuições específicas da indústria petrolífera no desenvolvimento humano. Segundo, porque os recursos distribuídos provenientes 
das indenizações petrolíferas são relativamente recentes. Ou seja, não houve tempo suficiente para que seu incremento pudesse ser convertido em sensíveis melhorias, visto que as demandas sociais são elevadas ${ }^{22}$.

$\mathrm{Na}$ análise do IQM-SF, Quissamã e Carapebus apresentaram melhores desempenhos em relação ao restante, destacando-se pelo aporte de recursos provenientes de royalties. No caso de Quissamã, 80,0\% da sua receita corrente líquida (RCL) eram provenientes de transferências de recursos (47,2\% dos royalties e 32,6\% do Imposto sobre Circulação de Mercadorias e Serviços, ocasionando a maior RCL per capita dentre todos os municípios. Possuía também a quarta maior capacidade de investimento do Estado do RJ e apresentava baixo comprometimento da receita com despesas de pessoal. Esse quadro extremamente favorável levou esse município, de pequeno porte, a ocupar o primeiro lugar no ranking de sustentabilidade fiscal. Em Campos dos Goytacazes, Casimiro de Abreu, Macaé e Rio das Ostras também se observaram a presença de royalties elevados, os que os distinguem dos demais. Além disso, por serem municípios de médio porte populacional, suas receitas de fundo de participação dos municípios por habitante são relativamente altas, gerando uma elevada capacidade potencial de gasto ${ }^{25}$.

Em relação ao IQM-PD, Macaé apresentou melhor potencial para desenvolvimento nos dois anos de análise. Esse pólo possui toda a infraestrutura básica para a atividade extrativa da Bacia de Campos. Recebeu uma grande quantidade de empresas produtoras de bens e de serviços, atraindo um grande fluxo demográfico. Criou demanda de mãode-obra especializada, estimulando a criação de cursos técnicos de níveis médio e superior, inclusive em outros municípios, destacando-se Campos dos Goytacazes, onde também se desenvolvem atividades de suporte aos investimentos petrolíferos. Entretanto, muitos trabalhadores com emprego formal em Macaé, em busca de local mais tranquilo e aprazível para viver, passaram a residir em outros municípios da região, como Campos dos Goytacazes,
Rio das Ostras, Santa Maria Madalena e Conceição de $\mathrm{Macabu}^{26}$.

O elevado crescimento demográfico de alguns municípios da MRA-5 foi influenciado pelas atividades extrativistas, que além de atraírem mão-de-obra qualificada para ocupar seus postos de trabalho, trouxeram milhares de pessoas de diversas partes do Estado do Rio de Janeiro e do país, com pouca ou nenhuma qualificação para trabalhar num setor de alta tecnologia, incapaz de absorver esse tipo de demanda. Isso contribuiu para a favelização, a informalidade e o aumento da violência, principalmente em Macaé e Rio das Ostras que vêm apresentando as maiores taxas de crescimento populacional da área de estudo ${ }^{27}$.

Caso semelhante foi encontrado em um estudo realizado na Província Petrolífera de Urucu, no município de Coari, Amazonas, onde, devido a uma conjuntura política einstitucional, essa exploração econômica, indiretamente, se tornou mais um elemento causador de processos migratórios, de assentamentos precários e de desenraizamentos de comunidades rurais de seus sítios de origem. A multidão migrante que se fixou, de forma crescente e contínua, e ainda se fixa nas periferias da sede de Coari, muitas vezes em condições subumanas, aumenta de forma exponencial, a insalubridade já existente ${ }^{28}$. Outro exemplo foi encontrado também na Venezuela, onde a crise do modelo de sociedade exportadora de petróleo levou o país a uma taxa de homicídios próxima aos 500 mortos por cem mil habitantes, colocando-se entre os países mais violentos da América ${ }^{29}$.

No IQM-C, as localidades mais carentes são aquelas que não conseguiram universalizar os benefícios sociais decorrentes de seu crescimento econômico. Grande parte é, ainda, de base agrícola. Já os menos carentes são aqueles cuja base econômica repousa nas atividades industriais, de comércio e/ou serviços e que apresentam as maiores populações, como Macaé e Campos dos Goytacazes ${ }^{4,15}$.

Através da observação de habitações e bairros, é possível identificar a grande desigualdade existente. A população 
carente se mantém numa rotina degradante e insustentável, com a falta de serviços públicos básicos e com as condições precárias em que vivem. Reflexo da condição social e econômica de seus moradores, associada à falta de políticas públicas, a qualidade da habitação para onde retornam os brasileiros após a jornada diária de trabalho ou estudo está diretamente ligada à sua qualidade de vida como um todo $^{30}$.

Apesar de mostrarem as potencialidades dos municípios de maneira mais próxima da realidade, a utilização dos índices compostos, como instrumento de avaliação da efetividade social das políticas públicas ou como instrumento de alocação prioritária do gasto social está sujeita a fortes questionamentos ${ }^{31}$. Há questionamentos acerca do grau de arbitrariedade com que se definem os pesos com os quais os indicadores devem ser ponderados no cômputo da medida final. Há ainda críticas com relação às distorções na seleção de públicos-alvo a que o uso desses indicadores sintéticos podem levar, sobretudo em casos de programas setoriais ${ }^{32}$. Cabe salientar que, embora a ausência de informações simultâneas para todos os indicadores seja uma grande limitação, o estudo buscou realizar uma análise exploratória compatível com análises de plausabilidade propostas para a avaliação de intervenções em Saúde Pública por Habicht et al., em que a coerência dos resultados pode reforçar a plausibilidade, não procedendo, portanto, questões de validade, mas indicadas quando o objetivo é o cálculo de medidas de associação ${ }^{33}$.

Em relação ao IES, Macaé e Nova Friburgo ocuparam o $1^{\circ}$ e $2^{\circ}$ lugares no ranking da MRA- 5 e o $5^{\circ}$ e $7^{\circ}$, no do Estado do RJ, respectivamente. Apesar das melhores situações sociais, esses municípios tiveram um dos piores resultados no índice de violência, que é uma das dimensões do IES. Nesse caso, os representantes da ZPP e da ZLZPP encontravam-se na $10^{\mathrm{a}} \mathrm{e} 7^{\mathrm{a}}$ posições no ranking da MRA-5, e na $75^{\text {a }}$ e $55^{\text {a }}$, no do Estado do RJ, simultaneamente $^{34}$.
O desenvolvimento sustentável é um novo paradigma que situa o ser humano como centro do processo de desenvolvimento, considerando o crescimento econômico como um meio e não como um fim. Esse paradigma tem por meta proteger as oportunidades de vida das gerações futuras e respeitar a integridade dos sistemas naturais que possibilitam a existência de vida na Terra $^{19}$

Provavelmente, em algum momento próximo, a produção de petróleo atingirá o seu pico máximo de exploração e produção, passando, logo em seguida, a um período de declínio. Esse acontecimento provocará não somente uma elevação no preço do petróleo, como muitos bens e serviços por ele produzidos. Desse modo, pensando no desenvolvimento sustentável e evitando grandes impactos referentes ao declínio, torna-se imprescindível a estimulação de um processo de crescimento estável, buscando a utilização de energias renováveis, distribuição equitativa de renda, diminuindo as diferenças sociais e que gerem menos impactos à população e ao planeta ${ }^{18,35}$.

É importante discutir a sustentabilidade na dimensão econômica e política garantindo a alocação e distribuição eficiente dos recursos naturais dentro de uma escala apropriada, não deixando de lado o fortalecimento de processos democráticos voltados ao enfrentamento de desafios presentes e futuros ${ }^{18}$.

OBrasil deve buscar experiências exitosas que possam ser adaptadas à realidade do país e que visem a aplicação dos recursos oriundos do petróleo de forma mais equânime, principalmente após a descoberta do présal. O modelo norueguês é visto por vários economistas como a referência de excelência, em 1990 o país criou o Fundo Petrolífero Estatal Norueguês (FPEN). Nesse fundo, a União tem a posse de $100 \%$ das receitas do petróleo. O contrário do que acontece no Brasil, onde $50 \%$ da receita ficam nas mãos da União. Esse fundo tem como objetivos promover a estabilidade macroeconômica e garantir rentabilidades futuras para a população aplicando na poupança. A Noruega 
aplica todo o recurso que recebe da atividade petrolífera em um fundo de pensão. $\mathrm{O}$ dinheiro é investido no exterior, na compra de ações e bônus, os dividendos das aplicações é que são gastos no país ${ }^{36}$.

No entanto, tal exemplo não pode ser aplicado por completo no Brasil, já que o país necessita de investimentos em curto prazo, não devendo, por esse motivo, toda a sua receita do petróleo com títulos e ações no exterior, pois países em desenvolvimento necessitam de mais investimentos em infraestrutura, educação, saneamento, saúde, habitação popular, dentre outros. O Brasil deverá buscar a sustentabilidade da dinâmica econômica pós-ciclo do petróleo em outras atividades econômicas que substituam a atividade petrolífera ${ }^{36}$.

Outro caso relevante é da Venezuela. Apesar de ter grandes reservas, os recursos provenientes do petróleo não trouxeram a estabilidade macroeconômica para o país e a atividade de extração e produção permanece sendo a base da economia. Visando a sustentabilidade econômica, a Venezuela tem buscado investir grande parte das receitas em infraestrutura e investimentos sociais. Esse caminho escolhido pelo país vem gerando resultados surpreendentes. Caso a experiência venezuelana mostrar sinais de sucesso nos próximos períodos, essa será de grande importância para a definição do modelo brasileiro, já que as realidades socioeconômicas são bem parecidas ${ }^{36}$.

\section{Conclusão}

Foi verificado um crescimento econômico excludente com grande dependência dos royalties e participações especiais, nos municípios da MRA-5, principalmente nos da ZPP. Diante desse panorama, torna-se necessário uma abordagem aos problemas do desenvolvimento, de maneira sistêmica, interdisciplinar e intersetorial, em todos os níveis de organização social ${ }^{37,38,39}$. Para tal, é necessária a implantação de mecanismos de participação popular como forma de legitimar a gestão e racionalizar o uso dos recursos públicos, principalmente no que concerne aos royalties do petróleo. Desse modo, será possível originar a médio e longo prazo uma cultura desenvolvimentista capaz de promover a continuidade de projetos, mesmo com as alternâncias de poder ${ }^{40}$.

Vale registrar que a não conversão da produção petrolífera em um enclave econômico depende diretamente da promoção de encadeamentos dinâmicos por outros ramos da indústria e serviços especializados, a fim de potencializar seu raio de alcance no que se refere aos efeitos multiplicadores. Assim, a indústria do petróleo possui fortes efeitos de encadeamento que podem deflagrar virtuoso processo de mudanças estruturais ou, ao contrário, permanecer como mero "enclave" regional.

\section{Referências}

1. Borba RC, Oliveira VM, Silva Neto R. A influência do petróleo na dinâmica econômica das cidades: um estudo comparativo entre Macaé (Brasil) e Aberdeen (Reino Unido). II Jornada Nacional da Produção Científica em Educação Profissional e Tecnológica São Luís (MA), 2007.

2. Mariano JB. Impactos ambientais do refino do petróleo [Dissertação de Mestrado]. Rio de Janeiro: Universidade Federal do Rio de Janeiro; 2001.

3. Goldemberg J, Moreira JR. Política Energética no Brasil. Estudos Avançados. 2005;19(55):215-28.

4. Pacheco CAG. A aplicação e os impactos dos royalties do petróleo no desenvolvimento econômico dos municípios confrontantes da Bacia de Campos [Monografia de
Bacharelado]. Rio de Janeiro. Universidade Federal do Rio de Janeiro: Instituto de Economia; 2003.

5. Silvestre BS, Dalcol PRT. Geographical proximity and innovation: Evidences from the Campos Basin oil \& gas industrial agglomeration — Brazil. Technovation. 2009;29:546-561.

6. Moura ANR, Cosenza HJSR. Os royalties do petróleo como potencializadores de desenvolvimento sustentável: o caso do município de Cabo Frio. VI conferencia Regional de ISTR para América Latina y el Caribe. 8 al 11 noviembre de 2007, Salvador de Bahia, Brasil. Organizan: ISTR y CIAGS/UFBA.

7. Barbosa DH (coord.). Guia dos royalties do petróleo e do gás natural. Agência Nacional do Petróleo (ANP). Rio de Janeiro: ANP; 2001. 
8. Fernandes CF. A evolução da arrecadação de royalties do petróleo no Brasil e seu impacto sobre o desenvolvimento econômico do Estado do Rio de Janeiro [Monografia de Bacharelado]. Rio de Janeiro. Universidade Federal do Rio de Janeiro: Instituto de Economia; 2007.

9. Valdivia G. Governing relations between people and things: Citizenship, territory, and the political economy of petroleum in Ecuador. Political Geography. 2008;27(4):456-77.

10. Silva JMC, Bozelli RL, Santos LF, Lopes AF. Impactos Ambientais da Exploração e Produção de Petróleo na Bacia de Campos - Rio de Janeiro. IV Encontro Nacional da Anppas. 4, 5 e 6 de junho de 2008. Brasília (DF), Brasil.

11. Alonso SG, Esteban-Hernández J, Rivera YV, HernándezBarrera V, Miguel AG. Contaminación del agua en Fuentes cercanas a campos petrolíferos de Bolivia. Rev Panam Salud Publica. 2010;28(4):235-43.

12. Ministério da Ciência e Tecnologia. Conselho Nacional de Desenvolvimento Científico e Tecnológico. Termo de referência da Macro-Região Ambiental Cinco (MRA-5) - Área de exploração de petróleo da Bacia de Campos. [Edital MCT-CNPq/MS-SCTIE-DECIT/CT - Saúde Número 24/2006 - Anexo VII] [internet]. Brasil; 2006. [citado 15 maio 2007]. Disponível em: <http://memoria. cnpq.br/servicos/editais/ct/2006/edital_0242006_ anexo7.pdf $>$.

13. Governo do Estado do Rio de Janeiro. Secretaria de Planejamento e Gestão. Centro de Informações e Dados do Rio de Janeiro. Índice de Qualidade dos Municípios Sustentabilidade Fiscal - CD-ROM, Rio de Janeiro: CIDE; 2002.

14. Governo do Estado do Rio de Janeiro. Secretaria de Planejamento e Gestão. Centro de Informações e Dados do Rio de Janeiro. Índice de Qualidade dos Municípios - Potencial para o Desenvolvimento - CD-ROM, Rio de Janeiro: CIDE; 2005.

15. Governo do Estado do Rio de Janeiro. Secretaria de Planejamento e Gestão. Centro de Informações e Dados do Rio de Janeiro. Índice de Qualidade dos Municípios Carências. Rio de Janeiro: CIDE; 2001a.

16. Governo do Estado do Rio de Janeiro. Secretaria de Planejamento e Gestão. Centro de Informações e Dados do Rio de Janeiro. Índice de qualidade dos municípios: necessidades habitacionais (IQM - Necessidades Habitacionais). Rio de Janeiro: CIDE; 2001.

17. Ministério do Planejamento, Orçamento e Gestão. Instituto Brasileiro de Geografia e Estatística. Censos (1980, 1991, 2000), Contagem (1996) e Projeções Intercensitárias (1981-2007), segundo faixa etária, sexo e situação de domicílio [internet]. Brasil; 1991,1995. [citado 15 maio 2007]. Disponível em: <http://tabnet.datasus.gov.br/cgi/ deftohtm.exe?ibge/cnv/popRJ.def $>$.

18. Santos M, Strauch J, Ajara C. Análise da sustentabilidade das zonas geoeconômicas do estado do Rio de Janeiro. Petróleo, royalties e região ano VIII, nº 29. Campos dos Goytacazes/ RJ. Set 2010.
19. Aquino CNP. Um estudo dos royalties de petróleo: impactos sobre indicadores sociais nos municípios do Rio de Janeiro [Dissertação de Mestrado]. Rio de Janeiro: Universidade Cândido Mendes; 2004.

20. Postali F. Efeitos da distribuição de royalties do petróleo sobre o crescimento dos Municípios no Brasil [internet]. [citado 5 jul 2008]. Departamento de Economia, Universidade de São Paulo, Anpec 2007. Disponível em: <www.anpec.org.br/encontro2007/artigos/A07A072.pdf>.

21. Honorato C (coord.). Análise comparativa da gestão de recursos públicos da região dos municípios produtores de petróleo do Estado do Rio de Janeiro. Rio de Janeiro: OVERJ/ ROLAC UN-HABITAT; 2005.

22. Souto A. Como reconhecer um bom governo? O papel das administrações municipais na melhoria da qualidade de vida. São Paulo: Publicações Pólis; 1995. p.21.

23. Guimarães JRS, Jannuzzi PM. IDH, Indicadores Sintéticos e suas aplicações em políticas públicas: uma análise crítica. $\mathrm{R}$ B Estudos Urbanos e Regionais. 2005;7(1).

24. Guimarães RP, Feichas SAQ. Desafios na construção de indicadores de sustentabilidade. Ambiente e sociedade 2009;XII(2):307-23.

25. Governo do Estado do Rio de Janeiro. Secretaria de Planejamento e Gestão. Centro de Informações e Dados do Rio de Janeiro. Índice de Qualidade dos Municípios Sustentabilidade Fiscal - CD-ROM, Rio de Janeiro: CIDE; 2002.

26. Governo do Estado do Rio de Janeiro. Secretaria de Planejamento e Gestão. Centro de Informações e Dados do Rio de Janeiro. Índice de Qualidade dos Municípios - Potencial para o Desenvolvimento - CD-ROM, Rio de Janeiro: CIDE; 2005.

27. Barral Neto J, Silva Neto R. Reestruturação produtiva e interiorização da economia no Estado do Rio de Janeiro: Uma nova dinâmica para a Região Norte Fluminense. In: XV Encontro Nacional de Estudos Populacionais, ABEP. Minas Gerais: Caxambu. Brasil; 2006.

28. Almeida WS, Souza NM. Coari: petróleo e sustentabilidade - um exemplo Amazônico. In: Desenvolvimento e Meio Ambiente. UFPR, 2008;17,69-92.

29. Briceño-León R. A violência na Venezuela: renda petroleira e crise política. Ciênc Saúde Coletiva. 2007;11(Suppl):1223-33.

30. Ramos MS. Habitação e saneamento: indicadores no contexto do petróleo. Revista Visões. 5a. Ed. 2008;5(1).

31. Ryten J. Should there be a human development index? Statistique, Développement et Droit de l'Homme Seminar, Montreaux, Setembre; 2000.

32. Jannuzzi PM. Indicadores para diagnóstico, monitoramento e avaliação de programas sociais no Brasil. Rev Serviço Público. 2005;56(2):137-60.

33. Habicht JP, Victora CG; Vaughan JP. Evaluation designs for adequancy, plausibility and probability of public health programme performance and impact. Int J Epidemiol. 1999; 28(1):10-18. 
34. Porchmann M, Amorim R, Guerra A, Moretto A, Fonseca A, Campos A, et al. Atlas da exclusão social no Brasil Dinâmica e manifestação territorial. São Paulo: Editora Cortez; 2003.

35. Frumkin H, Hess J, Vindigni S. Energy and public health: the challenge of peak petroleum. Public Health Reports. $2009 ; 124$.

36. Dias BCC. Impactos do petróleo no desenvolvimento sócio-econômico do Espírito Santo [Dissertação de Mestrado]. Vitória: Universidade Federal do Espírito Santo; 2008.

37. Postali FAS. Petroleum royalties and regional development in Brazil: The economic growth of recipient towns. Resources Policy. 2009;34:205-13.
38. Geller H, Schaeffer R, Szklo A, Tolmasquim M. Policies for advancing energy efficiency and renewable energy use in Brazil. Energy Policy. 2004;32(12)1437-50.

39. Gurgel AM, Medeiros ACLV, Alves PC, Silva JM, Gurgel IGD, Augusto LGS. Framework dos cenários de risco no contexto da implantação de uma refinaria de petróleo em Pernambuco. Ciênc Saúde Coletiva. 2009;14(6):2027-38.

40. Coelho AS. As participações governamentais e o impacto dos royalties sobre a economia do Estado do Rio de Janeiro [internet]. Jus Navigandi 2007; 1318. [citado 5 jun 2007]. Disponível em: < http://jus2.uol.com.br/doutrina/texto.asp?id=9476>.

Recebido em: 02/04/2010 Versão final apresentada em: 21/01/2011

Aprovado em: 28/02/2011 